\title{
An increasing threat in hospitals: multidrug-resistant Acinetobacter baumannii
}

\author{
Lenie Dijkshoorn*, Alexandr Nemec ${ }^{\ddagger}$ and Harald Seifert ${ }^{\S}$
}

Abstract | Since the 1970s, the spread of multidrug-resistant (MDR) Acinetobacter strains among critically ill, hospitalized patients, and subsequent epidemics, have become an increasing cause of concern. Reports of community-acquired Acinetobacter infections have also increased over the past decade. A recent manifestation of MDR Acinetobacter that has attracted public attention is its association with infections in severely injured soldiers. Here, we present an overview of the current knowledge of the genus Acinetobacter, with the emphasis on the clinically most important species, Acinetobacter baumannii.

DNA-DNA hybridization Determines the degree of similarity between the genomic DNA of two bacterial strains; the gold standard to assess whether organisms belong to the same species.

Pandrug-resistant In this Review, refers to A. baumannii that are resistant to all available systemic anti-A. baumannii antimicrobial agents, except for polymyxins.

* Department of Infectious Diseases C5-P, Leiden University Medical Centre, Albinusdreef 2, P.O. BOX 9600, 2300 RC Leiden, the Netherlands.

${ }^{\ddagger}$ Centre of Epidemiology and Microbiology, National Institute of Public Health Srobarova 48, 10042 Prague, Czech Republic. sInstitute for Medical Microbiology, Immunology and Hygiene, University of Cologne, Goldenfelsstrasse 19-21, 50935 Cologne, Germany.

Correspondence to L.D. e-mail: l.dijkshoorn@lumc.nl doi:10.1038/nrmicro 1789
Intensive care units (ICUs) of hospitals harbour critically ill patients who are extremely vulnerable to infections. These units, and their patients, provide a niche for opportunistic microorganisms that are generally harmless for healthy individuals but that are often highly resistant to antibiotics and can spread epidemically among patients. Infections by such organisms are difficult to treat and can lead to an increase in morbidity and mortality. Furthermore, their eradication from the hospital environment can require targeted measures, such as the isolation of patients and temporary closure or even reconstruction of wards. The presence of these organisms, therefore, poses both a medical and an organizational burden to health-care facilities.

One important group of bacteria that is associated with these problems is the heterogeneous group of organisms that belong to the genus Acinetobacter. This genus has a complex taxonomic history. Since the 1980s, in parallel with the emergence of acinetobacters as nosocomial pathogens, the taxonomy of the genus has been refined; 17 named species have been recognized and 15 genomic species (gen.sp.) have been delineated by DNA-DNA hybridization, but these do not yet have valid names (TABLE 1). The species that is most commonly involved in hospital infection is Acinetobacter baumannii, which causes a wide range of infections, including pneumonia and blood-stream infections. Numerous studies have reported the occurrence of multidrugresistant (MDR) A. baumannii in hospitals, and at some locations pandrug-resistant strains have been identified. Currently, A. baumannii ranks among the most important nosocomial pathogens. Additionally, the number of reports of community-acquired A. baumannii infection has been steadily increasing, although overall this type of infection remains rare. Despite the numerous publications that have commented on the epidemic spread of A. baumannii, little is known about the mechanisms that have favoured the evolution of this organism to multidrug resistance and epidemicity. In this Review, we discuss the current state of knowledge of the epidemiology, antimicrobial resistance and clinical significance of acinetobacters, with an emphasis on A. baumannii. The reader is also referred to previous reviews of this organism that have been written by pioneers in the field ${ }^{1,2}$.

\section{Identification of Acinetobacter species}

In 1986, a phenotypic system for the identification of Acinetobacter species was described ${ }^{3}$, which together with a subsequent simplified version ${ }^{4}$ has proven useful for the identification of most, but not all, Acinetobacter species. In particular, Acinetobacter calcoaceticus, A. baumannii, gen.sp. 3 and gen.sp. 13TU cannot be separated well by this system ${ }^{4}$. These species are also highly similar by DNA-DNA hybridization ${ }^{5}$ and it has therefore been proposed that they should be grouped together into the so-called A. calcoaceticus-A. baumannii (Acb) complex ${ }^{4}$. From a clinical perspective this might not be appropriate, as the complex combines three of the most clinically relevant species (A. baumannii, gen.sp. 3 and gen.sp. 13TU) with an environmental species (A. calcoaceticus). It is noteworthy that the performance of commercial systems for species identification that are used in diagnostic microbiology is also unsatisfactory. 
Using these systems, the clinically relevant species of the Acb complex are frequently uniformly identified as A. baumannii and many other species are not identified ${ }^{6-8}$. These problems have led to the development of genotypic methods for Acinetobacter species identification, some of which are discussed in BOX 1 (also see FIG. 1). Currently, precise species identification is not feasible in most laboratories, except for a few Acinetobacter reference laboratories. In light of the difficulties in distinguishing A. baumannii, gen.sp. 3 and gen.sp. 13TU, in this Review these species will be referred to as A. baumannii (in a broad sense) unless otherwise stated.

\section{Epidemiology of clinical acinetobacters}

The natural habitat of Acinetobacter species. Most Acinetobacter species have been found in clinical specimens (TABLE 1), but not all are considered to be clinically significant. One important question is where does A. baumannii come from? Furthermore, are there environmental or community reservoirs? As mentioned earlier, A. baumannii, gen.sp. 3 and gen.sp. 13TU are the most frequent species that are found in human clinical specimens ${ }^{5,9,10}$. Of these, gen.sp. 3 was the most prevalent species among clinical isolates in a Swedish study ${ }^{5}$. In 2 European studies, Acinetobacter lwoffii was the most

\section{Table 1 | Classification of the genus Acinetobacter}

\begin{tabular}{|c|c|c|}
\hline Species & Source & References \\
\hline \multicolumn{3}{|l|}{ Species that have valid names } \\
\hline Acinetobacter calcoaceticus & Soil and humans (including clinical specimens) & 3,5 \\
\hline Acinetobacter baumannii & $\begin{array}{l}\text { Humans (including clinical specimens), soil, meat and } \\
\text { vegetables }\end{array}$ & $3,5,17$ \\
\hline Acinetobacter haemolyticus & Humans (including clinical specimens) & 3,5 \\
\hline Acinetobacterjunii & Humans (including clinical specimens) & 3,5 \\
\hline Acinetobacter johnsonii & Humans (including clinical specimens) and animals & 3,5 \\
\hline Acinetobacter lwoffii (including gen.sp. 9) & Humans (including clinical specimens) and animals & 3,5 \\
\hline Acinetobacter radioresistens & Humans (including clinical specimens), soil and cotton & $3,5,105$ \\
\hline Acinetobacter ursingii & Humans (including clinical specimens) & 39 \\
\hline Acinetobacter schindleri & Humans (including clinical specimens) & 39 \\
\hline Acinetobacter parvus & Humans (including clinical specimens) and animals & 40 \\
\hline Acinetobacter baylyi & Activated sludge and soil & 100,106 \\
\hline Acinetobacter bouvetii & Activated sludge & 106 \\
\hline Acinetobacter towneri & Activated sludge & 106 \\
\hline Acinetobacter tandoii & Activated sludge & 106 \\
\hline Acinetobacter grimontii & Activated sludge & 106 \\
\hline Acinetobacter tjernbergiae & Activated sludge & 106 \\
\hline Acinetobacter gerneri & Activated sludge & 106 \\
\hline \multicolumn{3}{|l|}{ Species that have provisional designations } \\
\hline Acinetobacter venetianus & Sea water & 107 \\
\hline Gen.sp. 3 & Humans (including clinical specimens), soil and vegetables & $3,5,17$ \\
\hline Gen.sp. 6 & Humans (including clinical specimens) & 3,5 \\
\hline Gen.sp. 10 & Humans (including clinical specimens), soil and vegetables & $3,5,17$ \\
\hline Gen.sp. 11 & Humans (including clinical specimens) and animals & 3,5 \\
\hline Gen.sp. $13 \mathrm{BJ}$ or $14 \mathrm{TU}$ * & Humans (including clinical specimens) & 5,108 \\
\hline Gen.sp. 14BJ & Humans (including clinical specimens) & 108 \\
\hline Gen.sp. 15BJ & Humans (including clinical specimens) & 108 \\
\hline Gen.sp. 16 & Humans (including clinical specimens) and vegetables & 17,108 \\
\hline Gen.sp. 17 & Humans (including clinical specimens) and soil & 17,108 \\
\hline Gen.sp. 13TU & Humans (including clinical specimens) & 5 \\
\hline Gen.sp. 15TU & Humans (including clinical specimens) & 5 \\
\hline Gen.sp. 'between 1 and 3' & Humans (clinical specimens) & 9 \\
\hline Gen.sp. ‘close to 13TU’ & Humans (clinical specimens) & 9 \\
\hline
\end{tabular}




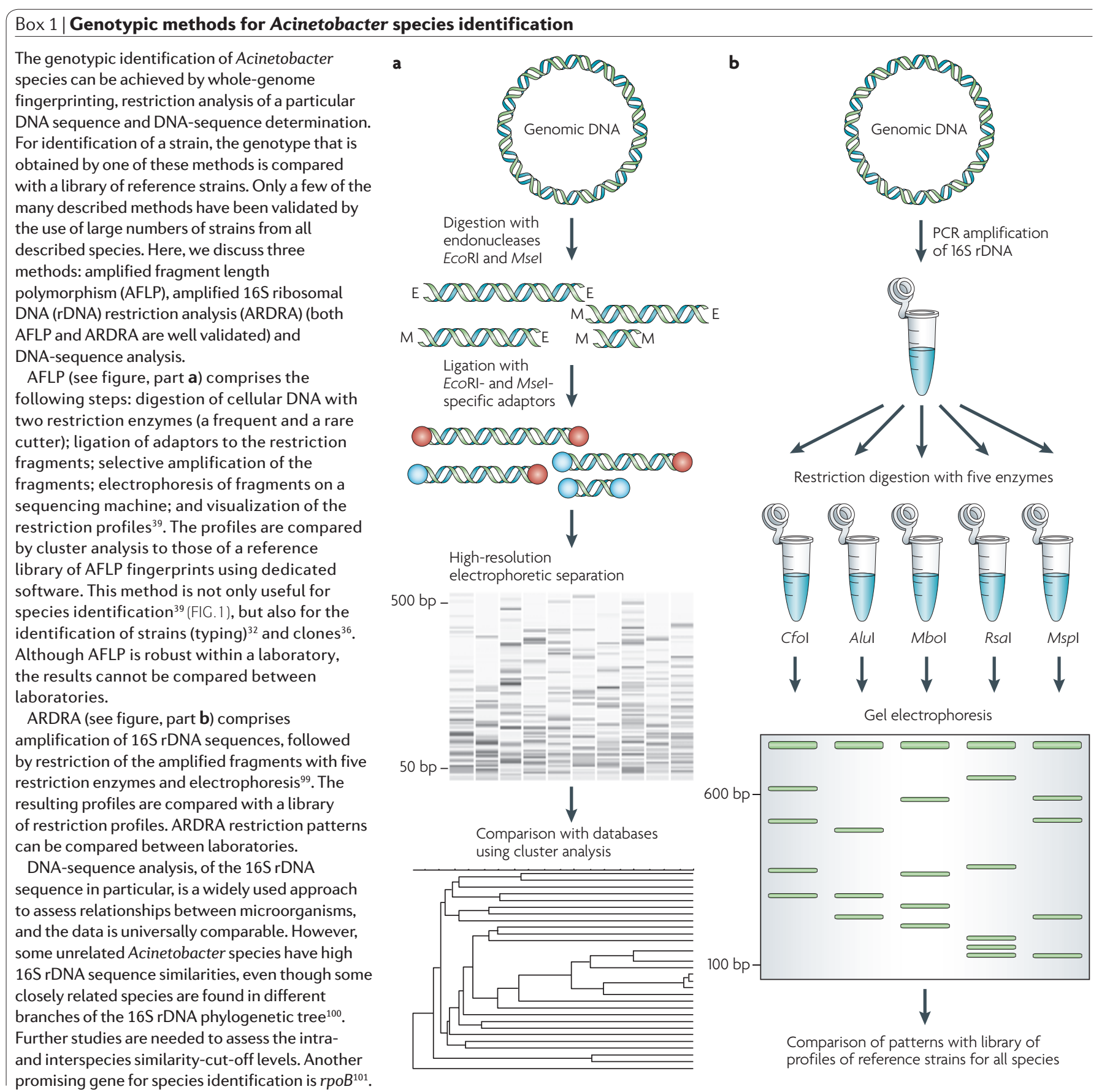

The genotypic identification of Acinetobacter species can be achieved by whole-genome fingerprinting, restriction analysis of a particular DNA sequence and DNA-sequence determination. For identification of a strain, the genotype that is DNA-sequence analysis.

AFLP (see figure, part a) comprises the sequencing machine; and visualization of the tibrary of AFLP fingerprints using dedicated species identification ${ }^{30} \mathrm{FC}$ 1), but also for the identification of strains (typing) ${ }^{32}$ and clones ${ }^{36}$. Although AFLP is robust within a laboratory, the results cannot be compared between laboratories.

ARDRA (see figure, part b) comprises amplification of 16S rDNA sequences, followed restriction enzymes and electrophoresis ${ }^{99}$. The can be compared between laboratories. DNA-sequence analysis, of the $16 \mathrm{~S}$ rDNA sequence in particular, is a widely used approach to assess relationships between microorganisms, and the data is universally comparable. However some unrelated Acinetobacter species have high 16S rDNA sequence similarities, even though some closely related species are found in different branches of the 16S rDNA phylogenetic tree ${ }^{100}$. and interspecies similarity-cut-off levels. Anothe promising gene for species identification is $r p o B^{101}$. a Digestion with
endonucleases EcoRl and Mse predominant species to be found on the skin of healthy individuals, with carrier rates of $29 \%$ and $58 \%$, whereas other Acinetobacter species, including Acinetobacter junii, Acinetobacter johnsonii, Acinetobacter radioresistens and gen.sp. 15BJ, were detected at lower frequencies ${ }^{11,12}$. The carrier rates for A. baumannii (including gen.sp. 13TU) in these studies ranged from 0.5 to $3 \%$, whereas for gen. sp. 3 the rates ranged from 2 to $6 \%{ }^{11,12}$. The faecal carriage of $A$. baumannii among non-hospitalized individuals in the United Kingdom and the Netherlands was $0.9 \%{ }^{13}$. The most predominant species in faecal samples from the Netherlands were A. johnsonii (17.5\%) and gen.sp. 11 $(4 \%)^{13}$. A. baumannii was also recovered from the body lice of homeless people ${ }^{14}$ and it was proposed that the organisms were associated with transient bacteraemia in these individuals. In a study in Hong Kong, the carrier rates of A. baumannii, gen.sp. 3 and gen.sp. 13TU on the skin of healthy individuals were 4,32 and $14 \%$, respectively ${ }^{15}$. Thus, the carrier rates for gen.sp. 3 and gen.sp. 13TU in that study were strikingly higher than in the European studies. These findings indicate that, at least in Europe, the carriage of A. baumannii in the community is relatively low. Apart from its occurrence in humans, A. baumannii has also been associated with infection and epidemic spread in animals at a veterinary clinic ${ }^{16}$. 


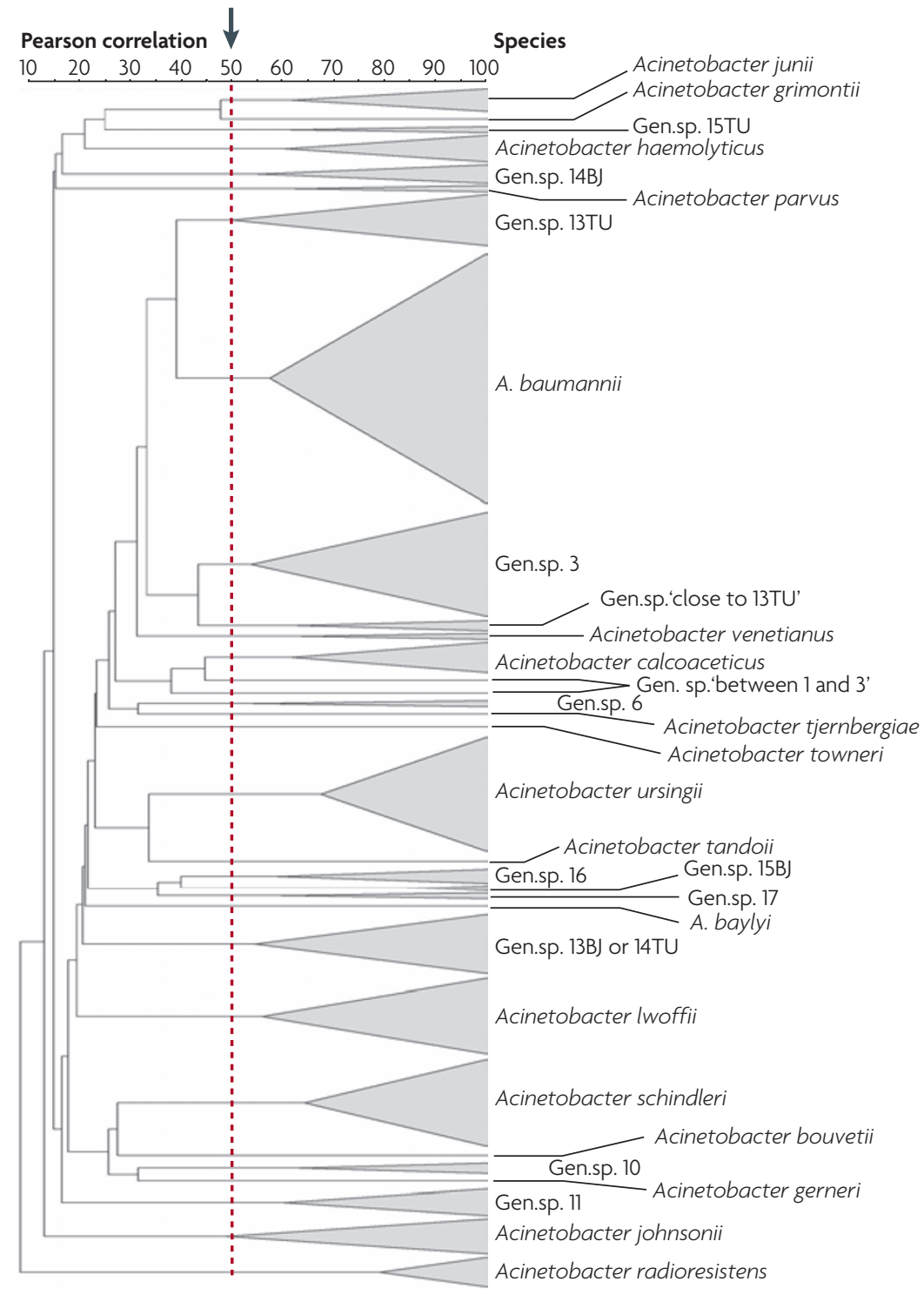

Figure 1 | Amplified fragment length polymorphism (AFLP) analysis of

Acinetobacter strains. A condensed dendrogram of the AFLP (described in BOX 1) fingerprints of 267 Acinetobacter reference strains of 32 described genomic species. All species are well separated at the $50 \%$ cluster-cut-off level, which emphasizes the power of this method for the delineation and identification of Acinetobacter species.

Endemic

The constant presence of an infectious agent in a given geographical area or hospital.
There are few available data on the environmental occurrence of A. baumannii, gen.sp. 3 and gen.sp. 13TU, but these species have been found in varying percentages in vegetables, fish, meat and soil ${ }^{17,18}$. A. baumannii has also recently been found in aquacultures of fish and shrimp farms in Southeast Asia ${ }^{19}$. However, it is not yet clear to what extent these findings are attributable to an environmental niche or to contact with humans or animals.

A. baumannii has been described as a soil organism, but without the support of appropriate references ${ }^{20}$. It was probably assumed that the wide occurrence of unspeciated acinetobacters in soil and water ${ }^{21}$ is also applicable to A. baumannii. However, in fact, there is little evidence that $A$. baumannii is a typical soil resident. Taken together, the existing data indicate that A. baumannii has a low prevalence in the community and that its occurrence in the environment is rare.

A. baumannii in hospitals. The most striking manifestation of A. baumannii is the endemic and epidemic occurrence of MDR strains in hospitals. The closely related gen.sp. 3 and gen.sp. 13TU might have a similar role ${ }^{22-24}$, and their involvement could have been underestimated as these species are phenotypically difficult to discriminate from A. baumannii. Most investigations of A. baumannii in hospitals have been ad hoc studies that were triggered by an outbreak. More in-depth studies of the prevalence of this species in hospitals, including antibiotic-resistant and antibiotic-susceptible strains, are required to better understand its true importance.

Depending on the local circumstances, and the strain in question, the pattern of an outbreak can vary. There can be a common source or multiple sources and some strains have a greater tendency for epidemic spread than others. Epidemiological typing - mostly by genotypic methods, such as amplified fragment length polymorphism (AFLP) analysis (BOX 1) - is an important tool that can distinguish an outbreak strain from other, concurrent strains, and assess the sources and mode of transmission of the outbreak strain.

A scheme that depicts the dynamics of epidemic A. baumannii on a hospital ward is provided in FIG. 2. An epidemic strain is most commonly introduced by a patient who is colonized. Once on a ward, the strain can then spread to other patients and their environment. A. baumannii can survive in dry conditions ${ }^{25}$ and during outbreaks has been recovered from various sites in the patients' environment, including bed curtains, furniture and hospital equipment ${ }^{26}$. These observations, and the success that cleaning and disinfecting patients' rooms has had in halting outbreaks, emphasize the role of the hospital environment as a reservoir for A. baumannii during outbreaks. The bacteria can be spread through the air over short distances in water droplets and in scales of skin from patients who are colonized ${ }^{27}$, but the most common mode of transmission is from the hands of hospital staff. Patients who are colonized or infected by a particular A. baumannii strain can carry this strain at different body sites for periods of days to weeks ${ }^{28}$, and colonization can go unnoticed if the epidemic strain is not detected in clinical specimens ${ }^{2,29}$.

Population studies of A. baumannii. Comparative typing of epidemic strains from different hospitals has indicated that there can be spread between hospitals. For example, during a period of outbreaks in the Netherlands that involved eight hospitals, one common strain was found in three of these hospitals and another common strain was found in two others ${ }^{26}$. Similar observations of interhospital spread of MDR strains in particular geographical areas have been made in the Czech Republic $^{30}$, the United Kingdom ${ }^{31}$, Portugal ${ }^{32}$ and the United States ${ }^{33}$.

Highly similar, but distinguishable, strains have been found at different locations and at different time points, 


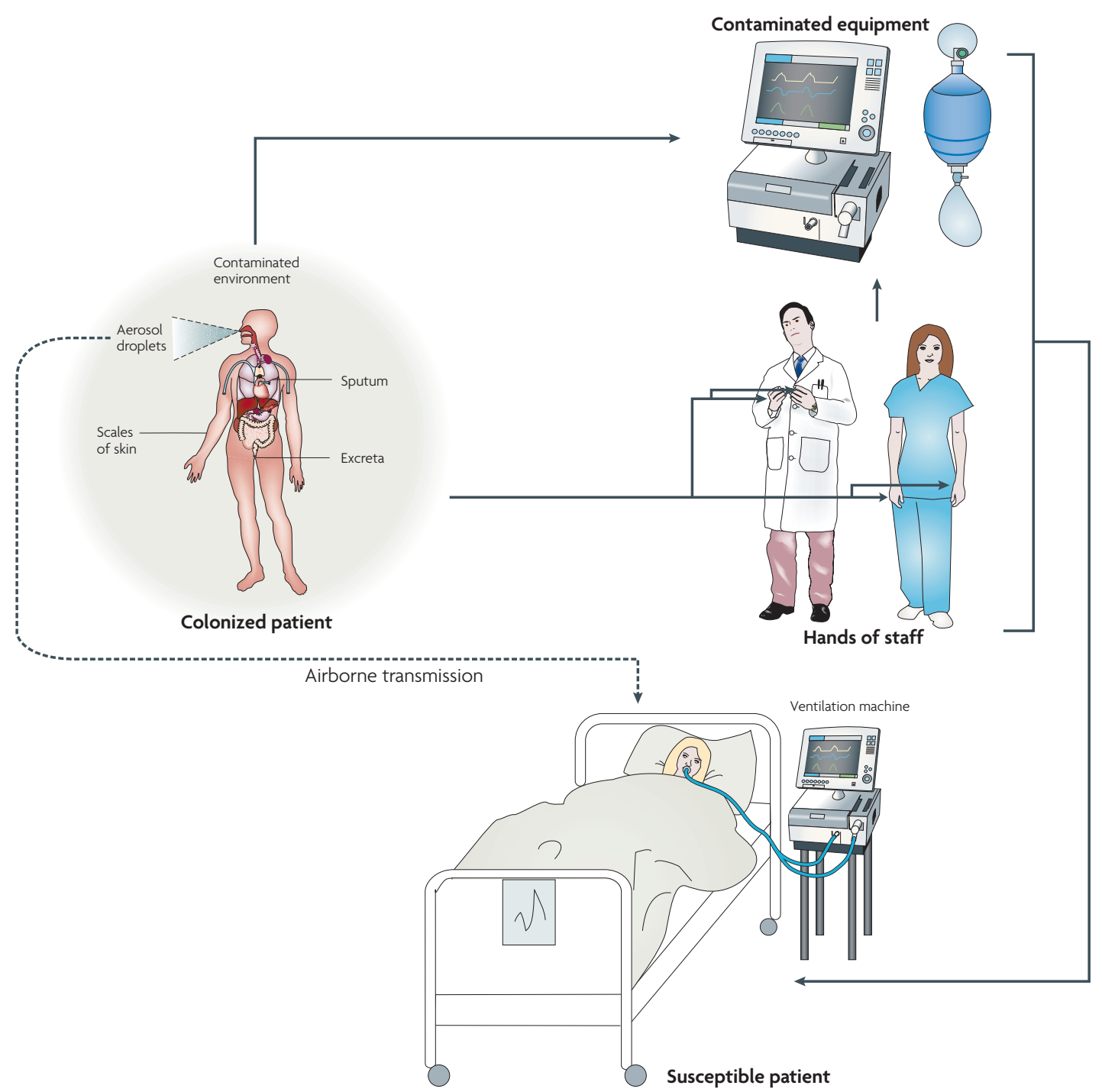

\section{Clones}

A group of bacteria that were isolated independently from different sources in time and space, but share so many identical traits that it is likely that they evolved from a common ancestor.

\section{Typing method}

A tool that differentiates bacterial strains below the species level.

\section{Ribotyping}

A typing method in which chromosomal DNA is digested by restriction enzymes, fragments are separated by electrophoresis and, finally, particular fragments are detected by labelled rRNA probes to generate DNA-

banding patterns, which allows the differentiation of bacterial isolates.

Figure 2 | Overview of the dynamics between patients, bacteria and the hospital environment. The possible modes of Acinetobacter baumannii entry into a ward are shown. Entrance through a colonized patient is the most likely mode. However, introduction through contaminated materials (such as pillows ${ }^{104}$ ) has also been documented. Notably, introduction by healthy carriers is also conceivable, although it is not known whether the rare strains that circulate in the community have epidemic potential. Once on a ward, A. baumannii can spread from the colonized patient to the environment and other susceptible patients. The direct environment of the patient can become contaminated by excreta, air droplets and scales of skin. Interestingly, A. baumannii can survive well in the dry environment ${ }^{25}$, a feature it shares with staphylococci. Hence, the contaminated environment can become a reservoir from which the organism can spread. The acquisition of $A$. baumannii by susceptible patients can occur through various routes, of which the hands of hospital staff are thought to be the most common, although the precise mode of transmission is usually difficult to assess.

without a direct epidemiological link. It is assumed that these strains represent particular lineages of descent (clones). Examples are European clones I-III ${ }^{34-36}$, which have been delineated by a range of genotypic typing methods, such as AFLP analysis (BOX 1 a; FIG. 1), ribotyping, macrorestriction analysis by pulsed-field gel electrophoresis and, most recently, multilocus sequence typing (see both MLST systems in Further information). Strains that belong to these clones are usually highly resistant to antibiotics, although within a clone there can be variation in antibiotic susceptibility. Apparently, these clones are genetically stable strains that are particularly successful in the hospital environment and evolve slowly during their spread. Whether these strains have particular virulence attributes or an enhanced ability to colonize particular patients (discussed below) remains to be established. Their wide spread might be explained by the transfer of patients between hospitals and regions over the course of time, although in many cases there is no evidence for this. It is also possible that they circulate at low rates in the community and are able to expand in hospitals under selective pressure from antibiotics. So far, their resistance to antimicrobial agents is the only known selectively advantageous trait. 
Clinical impact of Acinetobacter infections

Nosocomial infections. Acinetobacters are opportunistic pathogens that have been implicated in various infections that mainly affect critically ill patients in ICUs. Hospital-acquired Acinetobacter spp. infections include: ventilator-associated pneumonia; skin and soft-tissue infections; wound infections; urinary-tract infections; secondary meningitis; and bloodstream infections. These infections are mainly attributed to A. baumannii, although gen.sp. 3 and gen.sp. 13TU have also been implicated. Nosocomial infections that are caused by other Acinetobacter species, such as A.johnsonii, A. junii, A. lwoffii, Acinetobacter parvus, A. radioresistens, Acinetobacter schindleri and Acinetobacter ursingii, are rare and are mainly restricted to catheter-related bloodstream infections ${ }^{8,37-40}$. These infections cause minimal mortality and their clinical course is usually benign, although life-threatening sepsis has been observed occasionally ${ }^{41}$. The rare outbreaks of some of these species (for example, A. junii) have been found to be related to contaminated infusion fluids ${ }^{41}$.

The risk factors that predispose individuals to the acquisition of, and infection with, A. baumannii are similar to those that have been identified for other MDR organisms. These include: host factors such as major surgery, major trauma (in particular, burn trauma) and prematurity in newborns; exposurerelated factors such as a previous stay in an ICU, the length of stay in a hospital or ICU, residence in a unit in which A. baumannii is endemic and exposure to contaminated medical equipment; and factors that are related to medical treatment such as mechanical ventilation, the presence of indwelling devices (such as intravascular catheters, urinary catheters and drainage tubes), the number of invasive procedures that are performed and previous antimicrobial therapy ${ }^{42}$. Risk factors that are specific for a particular setting have also been identified, such as the hydrotherapy that is used to treat burn patients and the pulsatile lavage treatment that is used for wound débridement ${ }^{43,44}$.

The most frequent clinical manifestations of nosocomial A. baumannii infection are ventilator-associated pneumonia and bloodstream infection, both of which are associated with considerable morbidity and mortality, which can be as high as $52 \%{ }^{45,46}$. Risk factors for a fatal outcome are severity-of-illness markers, an ultimately fatal underlying disease and septic shock at the onset of infection. Bacteraemic A. baumannii pneumonia has a particularly poor prognosis ${ }^{46}$. A characteristic clinical manifestation is cerebrospinalshunt-related meningitis, caused by $A$. baumannii in patients who have had neurosurgery ${ }^{47}$. Wound infections have been reported mainly in patients who have severe burns or trauma, for example, soldiers who have been injured during military operations ${ }^{43,48}$. Urinarytract infections related to indwelling urinary-tract catheters usually run a more benign clinical course and are more frequent in rehabilitation centres than in ICUs ${ }^{49}$.
The clinical impact of nosocomial A. baumannii infection has been a matter of continuing debate. Many studies report high overall mortality rates in patients that have A. baumannii bacteraemia or pneumonia ${ }^{45,46}$. However, A. baumannii mainly affects patients with severe underlying disease and a poor prognosis. It has therefore been argued that the mortality that is observed in patients with A. baumannii infections is caused by their underlying disease, rather than as a consequence of $A$. baumannii infection. In a case-control study, Blot and colleagues ${ }^{50}$ addressed whether $A$. baumannii contributes independently to mortality and concluded that A. baumannii bacteraemia is not associated with a significant increase in attributable mortality. Similar findings for A. baumannii pneumonia have been reported by Garnacho and colleagues ${ }^{51}$. By contrast, in recent reviews of matched cohort and case-control studies, Falagas and colleagues ${ }^{52,53}$ concluded that A. baumannii infection was associated with an increase in attributable mortality, ranging from 7.8 to $23 \%$. These contradictory conclusions show that the debate on the clinical impact of A. baumannii is still ongoing.

Community-acquired infections. A. baumannii is increasingly recognized as an uncommon but important cause of community-acquired pneumonia. Most of the reported cases have been associated with underlying conditions, such as alcoholism, smoking, chronic obstructive pulmonary disease and diabetes mellitus. Community-acquired A. baumannii pneumonia appears to be a unique clinical entity that has a high incidence of bacteraemia, a fulminant clinical course and a high mortality that ranges from 40 to $64 \%$. It has been observed almost exclusively in tropical climates, in particular in Southeast Asia and tropical Australia ${ }^{54,55}$. It is currently unclear, however, if host factors or particular virulence factors are responsible for these severe infections. Multidrug resistance in these organisms is uncommon ${ }^{55}$. Other manifestations of community-acquired A. baumannii infections are rare.

Infections associated with natural disasters and war casualties. A characteristic manifestation of nosocomial A. baumannii is wound infection that is associated with natural or man-made disasters, such as the Marmara earthquake that occurred in 1999 in Turkey, the 2002 Bali bombing and military operations ${ }^{48,56,57}$. A strikingly high number of deep-wound infections, burn-wound infections and osteomyelitis cases have been reported to be associated with repatriated casualties of the Iraq conflict ${ }^{48}$. Isolates often had multidrug resistance. Based on the common misconception that A. baumannii is ubiquitous, it has been argued that the organism might have been inoculated at the time of injury, either from previously colonized skin or from contaminated soil. However, recent data clearly indicate that contamination of the environment of field hospitals and infection transmission in health-care facilities have had a major role in the acquisition of A. baumannii ${ }^{58}$. 


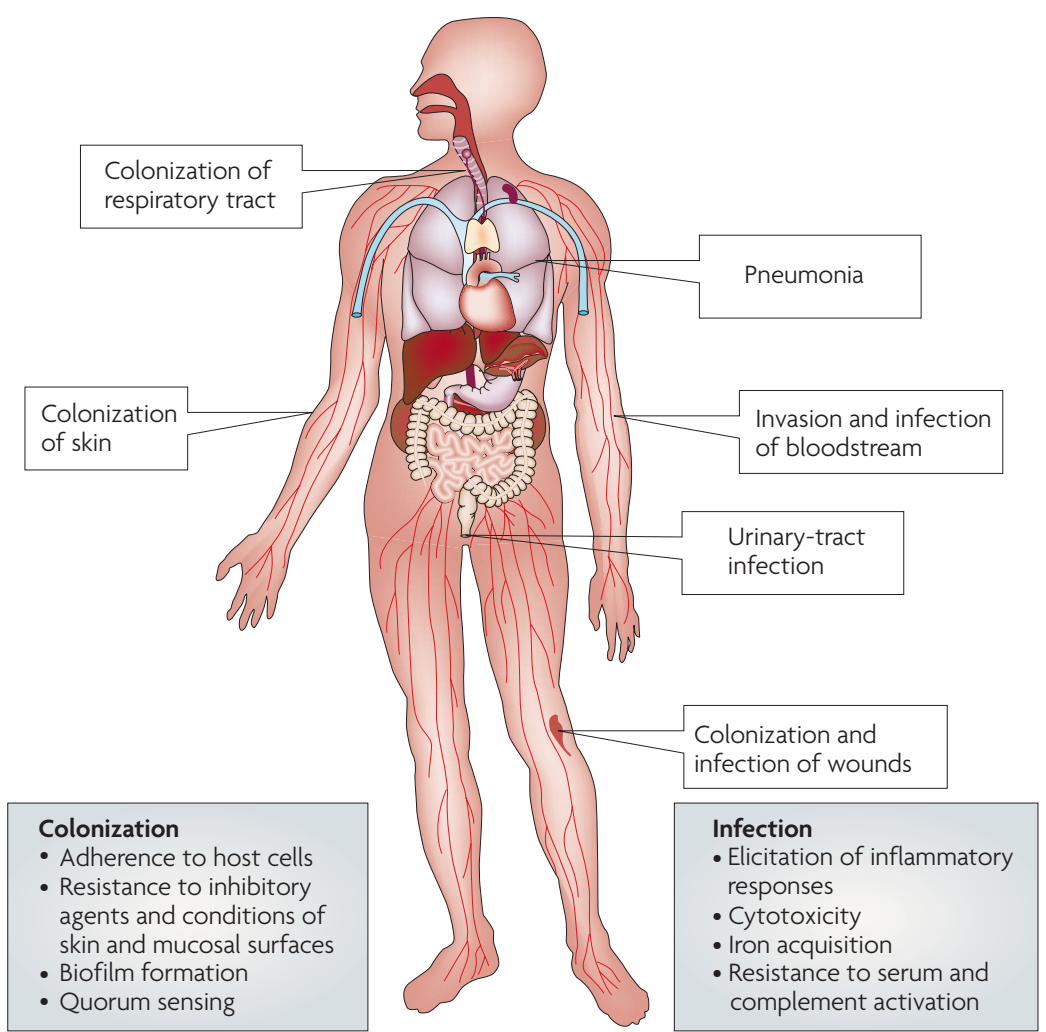

Environmental survival

- Resistance to desiccation, disinfectants and antibiotics

- Use of various substrates for growth

- Biofilm formation on surfaces,

equipment and devices

- Quorum sensing for regulation of,

for example, biofilm formation

Figure 3 | The factors that contribute to Acinetobacter baumannii environmental persistence and host infection and colonization. Adherence to host cells, as demonstrated in an in vitro model using bronchial epithelial cells ${ }^{62}$, is considered to be a first step in the colonization process. Survival and growth on host skin and mucosal surfaces require that the organisms can resist antibiotics and inhibitory agents and the conditions that are exerted by these surfaces. Outgrowth on mucosal surfaces and medical devices, such as intravascular catheters and endotracheal tubes ${ }^{61}$, can result in biofilm formation, which enhances the risk of infection of the bloodstream and airways. Quorum sensing ${ }^{59}$ might have a regulatory role in biofilm formation. Experimental studies have identified various factors that could have a role in A. baumannii infection, for example, lipopolysaccharide has been shown to elicit a proinflammatory response in animal models ${ }^{67,68}$. Furthermore, the A. baumannii outer membrane protein $\mathrm{A}$ has been demonstrated to cause cell death in vitro ${ }^{64}$. Iron-acquisition mechanisms ${ }^{65}$ and resistance to the bactericidal activity of human serum ${ }^{66}$ are considered to be important for survival in the blood during bloodstream infections. Environmental survival and growth require attributes such as resistance to desiccation ${ }^{25,60}$, versatility in growth requirements ${ }^{3}$, biofilmforming capacity ${ }^{61}$ and, probably, quorum-sensing activity ${ }^{59}$. Finally, adequate stressresponse mechanisms are thought to be required for adaptation to different conditions.

\footnotetext{
Quorum sensing

The phenomenon whereby the accumulation of signalling molecules enables a single cell to sense the number of bacteria (cell density) that are present, which allows bacteria to coordinate certain behaviours or actions.
}

\section{Epidemicity and pathogenicity}

The fact that colonization with A. baumannii is more common than infection, even in susceptible patients, emphasizes that the pathogenicity of this species is generally low. However, once an infection develops, it can be severe. Studies on the epidemicity and pathogenicity factors of A. baumannii are still at an elementary stage. A number of putative mechanisms that might have a role in colonization, infection and epidemic spread are summarized in FIG. 3. Genetic, molecular and experimental studies are required to elucidate these mechanisms in more detail.

Recent DNA sequencing of a single A. baumannii strain identified 16 genomic islands that carry putative virulence genes that are associated with, for example, cell-envelope biogenesis, antibiotic resistance, autoinducer production, pilus biogenesis and lipid metabolism $^{59}$. Resistance to desiccation, disinfectant ${ }^{25,60}$ and antibiotics is important for environmental survival. The extraordinary metabolic versatility ${ }^{3}$ of A. baumannii could contribute to its proliferation on a ward and in patients. Pilus-mediated biofilm formation on glass and plastics has been demonstrated ${ }^{61}$. If formed on medical devices, such as endotracheal tubes or intravascular catheters, these biofilms would probably provide a niche for the bacteria, from which they might colonize patients and give rise to respiratory-tract or bloodstream infections. Electron microscopy studies have demonstrated that pili on the surface of acinetobacters interact with human epithelial cells ${ }^{62}$. In addition, thread-like connections between these bacteria were suggestive of an early phase of biofilm formation. The pili and hydrophobic sugars in the O-side-chain moiety of lipopolysaccharide (LPS) ${ }^{63}$ might promote adherence to host cells as a first step in the colonization of patients. Quorum sensing, the presence of which has been inferred from the detection of a gene that is involved in autoinducer production ${ }^{59}$, could control the various metabolic processes, including biofilm formation.

Resistance to antibiotics, as well as the protective conditions of the skin (such as dryness, low $\mathrm{pH}$, the resident normal flora and toxic lipids) and those of the mucous membranes (such as the presence of mucus, lactoferrin and lactoperoxidase and the sloughing of cells) are prerequisites for bacterial survival in a host that is receiving antibiotics. In vitro and animal experiments have identified various factors that could have a role in A. baumannii infection. For example, A. baumannii outer membrane protein A (AbOmpA, previously called Omp38) has been associated with the induction of cytotoxicity ${ }^{64}$. Iron-acquisition mechanisms ${ }^{65}$ and serum resistance ${ }^{66}$ are attributes that enable the organism to survive in the bloodstream. The LPS and lipid A of one strain, at the time named A. calcoaceticus, had biological activities in animals that were similar to those of other enterobacteria ${ }^{67}$. These included lethal toxicity, pyrogenicity and mitogenicity for mouse-spleen B cells. More recently, A. baumannii LPS was found to be the major immunostimulatory component that leads to a proinflammatory response during A. baumannii pneumonia ${ }^{68}$ in a mouse model.

Taken together, the chain of events from environmental presence to the colonization and infection of patients demonstrates the extraordinary ability of A. baumannii to adapt to variable conditions. This ability suggests that the organism must possess, in addition to other factors, effective stress-response mechanisms. Together with its resistance to antibiotics, these mechanisms might explain the success of particular A. baumannii strains in hospitals. 
Antimicrobial susceptibility and treatment

Antimicrobial resistance. A. baumannii is attracting much attention owing to the increase in antimicrobial resistance and occurrence of strains that are resistant to virtually all available drugs ${ }^{69}$. This organism is generally intrinsically resistant to a number of commonly used antibiotics, including aminopenicillins, first- and secondgeneration cephalosporins and chloramphenicol ${ }^{70,71}$. It also has a remarkable capacity to acquire mechanisms that confer resistance to broad-spectrum $\beta$-lactams, aminoglycosides, fluoroquinolones and tetracyclines ${ }^{1}$. Numerous studies have suggested an upward trend in strains of A. baumannii that are resistant to these agents. However, because of the scarcity of large-scale surveillance studies from the 1970s to the 1990s and the difficulties in comparing local reports, such trends are difficult to quantify on a global level. Resistance rates can vary according to the country and the individual hospital, and depend on biological, epidemiological or methodical factors. For example, local susceptibility testing without correction for predominant MDR epidemic strains tends to overestimate the level of resistance ${ }^{72}$. It has also been shown that MDR isolates from geographically distant areas can be clonally related, whereas susceptible strains are genotypically heterogeneous $s^{30,34}$, which suggests that the problem of resistance might be associated with a limited number of successful A. baumannii lineages.

Despite the difficulties that are associated with estimating resistance trends, the potential of A. baumannii to develop resistance against virtually all available drugs is unquestionable (TABLE 2). Of particular concern is resistance to carbapenems - broad-spectrum $\beta$-lactams that were introduced by 1985 and that, for years, have been the most important agents for the treatment of infections caused by MDR A. baumannii. Although clinical $A$. baumannii isolates were shown to be invariably susceptible to these drugs in early studies ${ }^{70,71}$, hospital outbreaks caused by carbapenem-resistant strains had already been reported by the early $1990 \mathrm{~s}^{73}$ and, currently, the frequency of these strains in some areas can exceed $25 \%{ }^{74}$. Recently, resistance to polymyxins ${ }^{75}$ and tigecycline ${ }^{76}$ has also been described, which indicates that A. baumannii can cause infections that are fully refractory to the currently available antimicrobial armoury.

Resistance mechanisms. The resistance of A. baumannii to antimicrobial agents is mediated by all of the major resistance mechanisms that are known to occur in bacteria, including modification of target sites, enzymatic inactivation, active efflux and decreased influx of drugs (TABLE 2). $\beta$-lactamases are the most diverse group of enzymes that are associated with resistance, and more than 50 different enzymes, or their allelic forms, have been identified so far in A. baumannii. Aminoglycoside resistance has been attributed to at least nine distinct modifying enzymes, which can be found in different combinations in some strains ${ }^{77,78}$. Specific point mutations in the genes that encode DNA gyrase and topoisomerase IV have been correlated with resistance to fluoroquinolones $^{79}$, and resistance to tetracyclines has been associated with genes that encode tetracycline-specific efflux pumps ${ }^{80}$. Most genes that encode inactivating enzymes and specific efflux pumps are present only in some strains and are associated with genetic elements such as transposons, integrons or plasmids, which suggests they were acquired by horizontal transfer. Some of these genes are also common in other bacterial genera, whereas others are predominantly associated with the genus Acinetobacter (for example, the APH(3')-VI gene) or even with A. baumannii (for a list of genes that encode the OXA-type carbapenemases, see TABLE 2).

A few chromosomal resistance genes are present in most, if not all, A. baumannii strains. They are normally expressed at a low level but can be overexpressed as a result of genetic events. Chromosomal ADC-type $\beta$-lactamases can be upregulated as a consequence of the upstream insertion of an ISAbal sequence, which provides an efficient promoter ${ }^{81}$. This insertion sequence is widespread in $A$. baumannii and is thought to serve as a 'moving switch' to turn on those genes with which it is juxtaposed ${ }^{82}$. ISAbal is also thought to have a key role in some carbapenem-resistant strains by enhancing the expression of the intrinsic OXA-51-like carbapenemases $^{83}$. Another chromosomal system that is typical of A. baumannii is the AdeABC efflux system $^{84}$. Fully susceptible strains that contain the genes that encode $\mathrm{Ade} \mathrm{ABC}$ can spontaneously produce resistance mutations in the adeS or adeR genes, which regulate AdeABC expression ${ }^{85}$. Upregulation of AdeABC is so far the only mechanism that has been proven to decrease susceptibility to multiple antimicrobial classes in A. baumannii (TABLE 2).

The diversity of the determinants that confer resistance of $A$. baumannii to a particular group of antibiotics can best be illustrated by the mechanisms that are associated with carbapenem resistance ${ }^{86}$ (TABLE 2). These include metallo- $\beta$-lactamases (VIM-, IMP- and SIM-types), which have been reported worldwide and confer resistance to all $\beta$-lactams with the exception of monobactams. Nevertheless, the most widespread carbapenemases in A. baumannii are class $\mathrm{D} \beta$-lactamases. In addition to the intrinsic OXA-51-like enzymes, three unrelated groups of these carbapenem-hydrolysing oxacillinases have been distinguished, which are represented by OXA-23, -24 and -58 , respectively. Reduced susceptibility to carbapenems has also been associated with the modification of penicillin-binding proteins and porins or with upregulation of the AdeABC efflux system, and it has been suggested that the interplay of different mechanisms might result in high-level carbapenem resistance in A. baumannii ${ }^{87}$.

Despite the progress in the elucidation of the function and genetic basis of particular resistance mechanisms, knowledge of the genetic factors that contribute to multidrug resistance in A. baumannii is limited. Although resistance to multiple drugs can be associated with some epidemic lineages ${ }^{34,35}$, closely related MDR strains can differ greatly from each other in terms of the presence of particular resistance determinants and their combinations ${ }^{88}$. An important observation has recently been made by Fournier and colleagues ${ }^{20}$, who 


\section{Table 2 | Antimicrobial resistance mechanisms in Acinetobacter baumannii}

\begin{tabular}{|c|c|c|c|}
\hline $\begin{array}{l}\text { Mechanism or } \\
\text { responsible structure }\end{array}$ & Typical target drug & Note & References \\
\hline \multicolumn{4}{|l|}{$\beta$-lactam hydrolysis } \\
\hline$A D C-1,-3,-4,-6,-7$ & Cephalosporins & Chromosomal class $C(\mathrm{AmpC}) \beta$ Ls. Intrinsic to A. baumannii & \\
\hline TEM-1,-2 & Aminopenicillins & Narrow-spectrum class $A \beta L$ & 70,1 \\
\hline CARB-5* & Carboxypenicillins & Narrow-spectrum class $A \beta L$ & \\
\hline SCO-1 & Penicillins & Narrow-spectrum class A $\beta$ L. Plasmid encoded & \\
\hline $\begin{array}{l}\text { TEM-92, SHV-12, PER-1, } \\
\text { VEB-1 }\end{array}$ & $\begin{array}{l}\beta \text {-lactams, such as extended-spectrum } \\
\text { cephalosporins }\end{array}$ & $\begin{array}{l}\text { Extended-spectrum class A } \beta \text { Ls. Plasmid or chromosomal } \\
\text { genes flanked by insertion sequences }\end{array}$ & \\
\hline CTX-M-2 & Cefotaxime & Extended-spectrum class A $\beta$ Ls. Plasmid encoded & \\
\hline OXA-21,-37 & Oxacillins & Class D $\beta$ Ls. Class 1 integron-associated genes & 113,1 \\
\hline OXA-51-like & Carbapenems & Chromosomal class D $\beta$ Ls. Intrinsic to A. baumannii & \\
\hline OXA-23-like & Carbapenems & Class D $\beta$ Ls. Plasmid-encoded & \\
\hline OXA-24-like ${ }^{\ddagger}$ & Carbapenems & $\begin{array}{l}\text { Class D } \beta \text { Ls. Chromosomally encoded. Found in some epidemic } \\
\text { strains }\end{array}$ & \\
\hline OXA-58 & Carbapenems & $\begin{array}{l}\text { Class D } \beta \text { L. Transposon-borne gene on plasmids or } \\
\text { chromosome }\end{array}$ & \\
\hline IMP-1, $-2,-4,-5,-6,-11$ & Carbapenems & Class B metallo- $\beta$ Ls. Class 1 integron-associated genes & \\
\hline VIM-2, SIM-1 & Carbapenems & Class B metallo- $\beta$ Ls. Class 1 integron-associated genes & \\
\hline
\end{tabular}

Aminoglycoside modification

\begin{tabular}{|c|c|c|c|}
\hline AAC(3)-la & Gentamicin & Acetyltransferase. Class 1 integron-associated gene & 77,110 \\
\hline AAC(3)-Ila & Gentamicin, tobramycin & Acetyltransferase & 77 \\
\hline $\mathrm{AAC}\left(6^{\prime}\right)-\mathrm{Ib}$ & Tobramycin, amikacin & Acetyltransferase. Class 1 integron-associated gene & 77 \\
\hline $\mathrm{AAC}\left(6^{\prime}\right)-\mathrm{lh}, \mathrm{AAC}\left(6^{\prime}\right)-\mathrm{lad}$ & Tobramycin, amikacin & Acetyltransferase. Plasmid-encoded & 69,115 \\
\hline $\mathrm{APH}\left(3^{\prime}\right)-\mathrm{la}$ & Kanamycin & Narrow-spectrum phosphotransferase & 77,110 \\
\hline $\mathrm{APH}\left(3^{\prime}\right)-\mathrm{VI}$ & Amikacin, kanamycin & Phosphotransferase. Mostly plasmid encoded & 116 \\
\hline ANT(2")-la & Gentamicin, tobramycin & $\begin{array}{l}\text { Nucleotidyltransferase. Gene can be associated with class } 1 \\
\text { integron }\end{array}$ & 77 \\
\hline ANT( $\left.3^{\prime \prime}\right)-$ la & Streptomycin & Nucleotidyltransferase. Class 1 integron-associated gene & 77,110 \\
\hline \multicolumn{4}{|c|}{ Chloramphenicol modification } \\
\hline CAT1 & Chloramphenicol & Acetyltransferase. Plasmid or chromosomally encoded & 1 \\
\hline \multicolumn{4}{|l|}{ Target alteration } \\
\hline GyrA & Quinolones & Gyrase subunit. Mostly mutations in the Ser 83 codon & 79 \\
\hline ParC & Quinolones & $\begin{array}{l}\text { Topoisomerase IV subunit. Mostly mutations in the Ser } 80 \\
\text { codon }\end{array}$ & 79 \\
\hline ArmA & Aminoglycosides & 16 ribsomal RNA methylase. Plasmid encoded & 117 \\
\hline \multicolumn{4}{|l|}{ Active efflux } \\
\hline AdeABC & $\begin{array}{l}\text { Broad (for example, aminoglycosides } \\
\text { and quinolones) }\end{array}$ & $\begin{array}{l}\text { Chromosomally encoded RND-type pump. Present in most } \\
\text { A. baumannii strains }\end{array}$ & 84,85 \\
\hline AbeM & Broad (for example, quinolones) $)^{\S}$ & Chromosomally encoded MATE-type pump & 118 \\
\hline Tet(A) & Tetracycline & Found in most European clone I strains. Transposon borne & 80 \\
\hline Tet(B) & Minocycline, tetracycline & Found in most European clone II strains & 80 \\
\hline \multicolumn{4}{|c|}{ Changes in outer-membrane proteins (OMPs) } \\
\hline CarO & Carbapenems & 29-kDa OMP implicated in drug influx & 86 \\
\hline 33 to $36-k D a$ OMP & Carbapenems & $\begin{array}{l}\text { Other OMPs (OprD-like protein or 22-kDa OMP) have also } \\
\text { been associated with carbapenem resistance }\end{array}$ & 86 \\
\hline
\end{tabular}

The mechanisms listed have been studied in some detail in strains that have been identified as A. baumannii. Although some mechanisms are common in this species, others can be rare (for example, SCO-1 and SIM-1). In addition, the following resistance genes have been reported to occur in A. baumannii, although their contribution

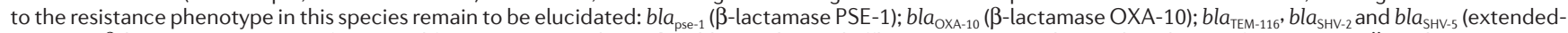
spectrum $\beta$-lactamases TEM-116, SHV-2 and SHV-5, respectively); cmlA (chloramphenicol efflux pump); strA and strB (phosphotransferases APH(3") and APH(6), respectively); $\operatorname{dhfr} 1$ and $d h f r X$ (dihydrofolate reductases); sul1 (dihydropteroate synthase); tet(M) (tetracycline ribosomal protection protein); and arr-2 (rifampicin ADPribosylating transferase). The genetic location of some resistance genes is based on a single observation and may not be their general property. Furthermore, genes that are associated with transposable elements may occur in different replicons. *Acinetobacter strain not identified to the species level. ${ }^{\star}$ Several allelic variants known. ${ }^{\S}$ As indicated by the expression of abeM cloned into Escherichia coli. $\beta \mathrm{L}, \beta$-lactamase. 


\section{Box 2 | Prevention and control of MDR A. baumannii in hospitals}

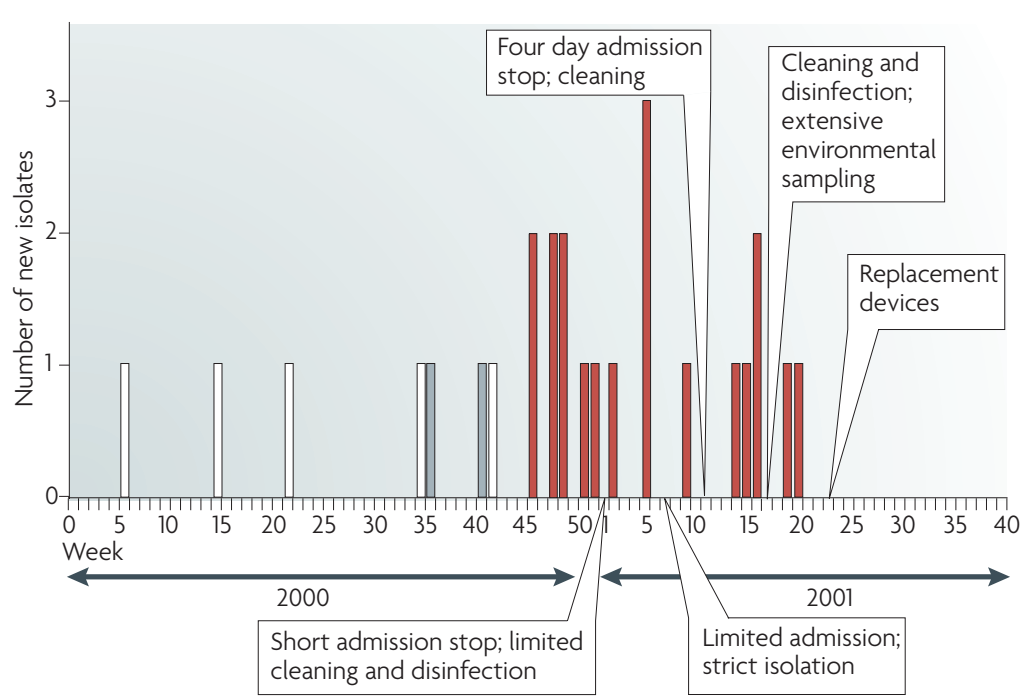

There is general agreement that every case of infection or colonization with multidrug-resistant (MDR) Acinetobacter baumannii should prompt enforcement of infection-control measures to prevent transmission. The containment strategies for A. baumannii are also largely applicable to those for meticillin-resistant

Staphylococcus aureus, which shows similar epidemiological behaviour. Infection control practices differ between countries. In the Netherlands, strict guidelines have been issued to prevent the transmission of highly resistant microorganisms such as MDR A. baumannii (WIP; see Further information). These imply there should be strict isolation of each patient who is infected by MDR A. baumannii, even in the absence of an outbreak. A typical example of the various measures that were taken to successfully combat an outbreak that occurred in a medical intensive care unit (ICU) at Leiden University Medical Center, the Netherlands ${ }^{102}$, is shown in the figure. The bars indicate the number of new cases per week (patients who were colonized or infected with A. baumannii). Outbreak strains (marked in red) were identified by molecular typing and distinguished from sporadic strains (marked in white and grey).

Outbreak management requires an active infection-control programme for patients and hospital personnel, including contact isolation to interrupt transmission as well as active surveillance cultures to detect $A$. baumannii-colonized patients ${ }^{26}$. Environmental surveillance cultures can identify contaminated surfaces in the patients' environments that might serve as secondary sources for $A$. baumannii transmission or a common source, such as a contaminated ventilator. Molecular typing of Acinetobacter isolates recovered from patients and environmental specimens is necessary to establish the magnitude of an outbreak and the possible modes of transmission. Combating large-scale outbreaks in the absence of a common source might require intensified measures, such as improved hand hygiene, cleaning and disinfection of the environment, cohort nursing, the isolation of patients in single rooms, the restriction of access to the ICU, appropriate antibiotic use, improving staffing ratios and, ultimately, closing a ward for cleaning and disinfection ${ }^{26,103}$. The eradication of an epidemic organism such as MDR A. baumannii from a ward is a burden to the hospital, both economically and logistically.

\section{Strict isolation}

Rigorous separation to prevent highly contagious or virulent agents that can spread both by air and contact.

Contact isolation

The separation of colonized or infected individuals from others to prevent, or limit, the direct or indirect transmission of diseases that are spread primarily by close or direct contact. compared the complete genomes of MDR (AYE) and susceptible (SDF) strains of A. baumannii. Whereas the AYE strain contained an 86-kilobase $(\mathrm{kb})$ genomic region, termed a resistance island (the largest identified in bacteria to date), in which 45 putative resistance genes were clustered, SDF exhibited a $20-\mathrm{kb}$ genomic island at the homologous location that comprised genes that encode transposases but no resistance genes. A region that is highly similar to the 20 -kb island has also been found in the genome of an A. baumannii strain that was isolated in 1951 (REF. 59). It is conceivable that such a genomic structure could serve as a 'hot spot' that facilitates the horizontal acquisition of resistance genes $^{20}$ and has had a crucial role in the development of $A$. baumannii multidrug resistance.

Options for treatment. Unfortunately, prospective controlled clinical trials for the treatment of A. baumannii infections have never been performed. Our knowledge of the best treatment options is therefore based on retrospective analysis of observational studies, small case series and in vitro data. Neither broad-spectrum penicillins nor cephalosporins are considered to be effective for the treatment of serious A. baumannii infections. Even if isolates are considered to be susceptible according to in vitro tests, their minimum inhibitory concentration values are usually close to the clinical breakpoint. Fluoroquinolones have remained active against sporadic $A$. baumannii strains but resistance is now widespread among epidemic A. baumannii strains, rendering these antimicrobial drugs no longer useful. Aminoglycosides, in particular tobramycin and amikacin, often retain their activity against resistant A. baumannii isolates; however, these compounds are rarely used alone and are more often applied in combination with other antimicrobials.

As A. baumannii is becoming increasingly resistant to carbapenems in hospitals worldwide, only a few treatment options remain. Sulbactam, a $\beta$-lactamase inhibitor that has unusual intrinsic activity against acinetobacters, has been used successfully for the treatment of MDR A. baumannii infections, such as meningitis, ventilator-associated pneumonia and catheter-related bacteraemia ${ }^{89,90}$. In most patients, sulbactam has been used in combination with ampicillin, but its usefulness is now also increasingly compromised by resistance.

In recent years, there has been a resurgence of the use of polymyxins for the treatment of MDR Gram-negative bacteria, in particular Pseudomonas aeruginosa and A. baumannii. An increasing body of evidence suggests that intravenous polymyxins can be used successfully for the treatment of ventilator-associated A. baumannii pneumonia, with less nephrotoxicity observed than had been anticipated ${ }^{91}$. Alternatively, these drugs can be applied topically by the use of an aerosol ${ }^{92}$. Resistance of A. baumannii against polymyxins is still extremely rare $^{75}$. Success rates of up to $60 \%$ have been reported, but the true efficacy of these antimicrobials remains to be shown in prospective studies.

Tigecycline was the first glycylcycline antibiotic to be launched and is one of the few new antimicrobials that has activity against Gram-negative bacteria that encompass not only most Enterobacteriaceae but also - at least in vitro - MDR A. baumannii. Several studies have tested the in vitro activity of tigecycline against A. baumannii and reported good bacteriostatic activity against strains that have a wild-type susceptibility profile, as well as those that are resistant to imipenem ${ }^{72}$. However, current evidence casts doubt on the role of tigecycline as a treatment for MDR A. baumannii infection, with reports of high tigecycline resistance ${ }^{93}$ and the occurrence of an A. baumannii bloodstream infection in a patient who received tigecycline $e^{76}$. 
Minimum inhibitory

concentration

The lowest concentration of an antibiotic that inhibits visible

growth of the organism.

Clinical breakpoint

A discriminatory antimicrobial

concentration that is used

to interpret the results of

susceptibility testing and to

define bacterial populations as

susceptible, intermediate or

resistant based on the

likelihood of therapeutic

success.
Antimicrobial combination therapy appears to be a reasonable alternative for the combat of MDR A. baumannii. A considerable number of in vitro studies, and animal studies using a mouse model of pneumonia, have been carried out to analyse the effect of combination therapy ${ }^{94-97}$. Suggested combinations include imipenem and amikacin; colistin and rifampin; and imipenem, rifampin and colistin. Clinical experience with combination therapy is limited. In the light of the few agents that are currently available to treat MDR A. baumannii infections, alternative treatment strategies and agents are urgently needed. A recent study has shown that, in an animal model, the lactoferrin-derived peptide $\mathrm{hlF}(1-11)$ is a promising candidate for the treatment of MDR A. baumannii ${ }^{98}$. However, new antimicrobials that have activity against $A$. baumannii are not expected to be available within the next decade.

\section{Conclusion and challenges}

Since the 1980s, the taxonomy of the genus Acinetobacter has undergone extensive changes, with the recognition of more than 30 gen.sp. The biological significance of most species other than those of the Acb complex remains to be elucidated owing to a lack of practical identification methods. This situation is expected to improve, however, in the near future with the introduction of well-validated sequence-based identification methods.

The occurrence of MDR and pandrug-resistant $A$. baumannii is a growing source of concern, and the exploration of new antibiotics, including host-defence peptides, is urgently required. Apart from MDR strains, there are many antibiotic-susceptible strains of A. baumannii, but their prevalence in hospitals and the community is unknown. It is a challenge to assess whether these strains have the potential to emerge to multidrug resistance (and epidemicity) once they enter an antibiotic-rich niche, such as a hospital. Basic questions remain to be answered about the role of the upregulation of resistance genes that are already present in the genome and the contribution of acquired resistance genes, including the possibility that they are stably integrated into the chromosome. It is also possible that the MDR strains that circulate in hospitals are distinct lineages or groups of lineages within A. baumannii, as has already been shown for strains that belong to European clones I-III. The recently developed MLST systems (see both MLST systems in Further information) are important tools that can be used to investigate the prevalence of particular strains at a global scale. However, these systems are based on the similarity of housekeeping genes and the genes that confer resistance and virulence could be located on mobile elements and clustered in genomic islands that are not linked to particular sequence types. Multi-strain genome analysis will probably provide some insight into these questions.

Little is known about the bacterial mechanisms that explain the success of A. baumannii in the hospital environment. Genomics, proteomics and fitness studies are expected to be helpful approaches to clarify the remarkable adaptability of the bacteria to variable conditions and selective pressures. Furthermore, apart from general host or treatment factors, there might be intrinsic host factors, such as a particular genetic constitution or innate immune status, that make a specific host susceptible to colonization and infection. Progress is also expected in this area.

The eradication of epidemic A. baumannii from the hospital environment demands a great effort (BOX 2). Some countries have managed to keep the microorganism at a sporadic level, with outbreaks occurring only occasionally. In many other countries, however, the organisms are endemic or epidemic. The combat of MDR A. baumannii (and other MDR organisms) reaches far beyond the hospital level and requires a combined strategy of decision makers and health-care officials, the challenge being to make hospitals a safer place for patients who are critically ill.
1. Bergogne-Bérézin, E. \& Towner, K. J. Acinetobacter spp. as nosocomial pathogens: microbiological, clinical, and epidemiological features. Clin. Microbiol. Rev. 9, 148-165 (1996).

Comprehensive review of the epidemiology, clinical significance, epidemiological typing and antibiotic resistance of acinetobacters.

2. Joly-Guillou, M. L. Clinical impact and pathogenicity of Acinetobacter. Clin. Microbiol. Infect. 11, 868-873 (2005).

3. Bouvet, P. J. M. \& Grimont, P. A. D. Taxonomy of the genus Acinetobacter with the recognition of Acinetobacter baumannii sp. nov., Acinetobacter haemolyticus sp. nov., Acinetobacter johnsonii sp. nov., and Acinetobacter junii sp. nov. and emended descriptions of Acinetobacter calcoaceticus and Acinetobacter Iwoffii. Int. J. Syst. Bacteriol. 36 , 228-240 (1986)

First study to split up the genus Acinetobacter into species.

4. Gerner-Smidt, P., Tjernberg, I. \& Ursing, J. Reliability of phenotypic tests for identification of Acinetobacter species. J. Clin. Microbiol. 29 277-282 (1991).

5. Tjernberg, I. \& Ursing, J. Clinical strains of Acinetobacter classified by DNA-DNA hybridization. APMIS 97, 595-605 (1989).

Confirmation of the taxonomy described in

reference 3 and demonstration of the high

similarity of $A$. calcoaceticus, $A$. baumannii and gen.sp. 3 and $13 T$ T.
6. Bernards, A. T. van der Toorn, J van Boven, C. P. \& Dijkshoorn, L. Evaluation of the ability of a commercial system to identify Acinetobacter genomic species. Eur. J. Clin. Microbiol. Infect. Dis. 15, 303-308 (1996)

7. van Dessel, H. et al. Outbreak of a susceptible strain of Acinetobacter species 13 (sensu Tjernberg and Ursing) in an adult neurosurgical intensive care unit. J. Hosp. Infect. 51, 89-95 (2002).

8 . Dortet, L., Legrand, P., Soussy, C. J \& Cattoir, V. Bacterial identification, clinical significance, and antimicrobial susceptibilities of Acinetobacter ursingii and Acinetobacter schindleri, two frequently misidentified opportunistic pathogens. J. Clin Microbiol 44, 4471-4478 (2006).

9. Gerner-Smidt, P. \& Tjernberg, I. Acinetobacter in Denmark: II. Molecular studies of the Acinetobacter calcoaceticus-Acinetobacter baumannii complex. APMIS 101, 826-832 (1993).

10. Seifert, H., Baginski, R., Schulze, A. \& Pulverer, C The distribution of Acinetobacter species in clinical culture materials. Zentralbl. Bakteriol. 279, 544-552 (1993).

11. Seifert, H. et al. Distribution of Acinetobacter species on human skin: comparison of phenotypic and genotypic identification methods. J. Clin. Microbiol. 35, 2819-2825 (1997)

12. Berlau, J., Aucken, H., Malnick, H. \& Pitt, T. Distribution of Acinetobacter species on skin of healthy humans. Eur. J. Clin. Microbiol. Infect. Dis. 18, 179-183 (1999).
13. Dijkshoorn, L. et al. Prevalence of Acinetobacter baumannii and other Acinetobacter spp. in faecal samples from non-hospitalised individuals. Clin. Microbiol. Infect. 11, 329-332 (2005).

14. La Scola, B. \& Raoult, D. Acinetobacter baumannii in human body louse. Emerg. Infect. Dis. 10, 1671-1673 (2004).

15. Chu, Y. W. et al. Skin carriage of acinetobacters in Hong Kong. J. Clin. Microbiol. 37, 2962-2967 (1999).

16. Boerlin, P., Eugster, S., Gaschen, F., Straub, R. \& Schawalder, P. Transmission of opportunistic pathogens in a veterinary teaching hospital. Vet. Microbiol. 82, 347-359 (2001).

17. Houang, E. T. et al. Epidemiology and infection control implications of Acinetobacter spp. in Hong Kong. J. Clin. Microbiol. 39, 228-234 (2001).

18. Berlau, J., Aucken, H. M., Houang, E. \& Pitt, T. L. Isolation of Acinetobacter spp. including A. baumannii from vegetables: implications for hospital-acquired infections. J. Hosp. Infect. 42, 201-204 (1999).

19. Huys, G. et al. Biodiversity of chloramphenicolresistant mesophilic heterotrophs from Southeast Asian aquaculture environments. Res. Microbiol.158, 228-235 (2007).

20. Fournier, P. E. et al. Comparative genomics of multidrug resistance in Acinetobacter baumannii. PLoS Genet. 2, e7 (2006). Description of an 86-kb genomic region that was termed a resistance island.

21. Baumann, P. Isolation of Acinetobacter from soil and water. J. Bacteriol. 96, 39-42 (1968). 
22. Seifert, H. \& Gerner-Smidt, P. Comparison of ribotyping and pulsed-field gel electrophoresis for molecular typing of Acinetobacter isolates. J. Clin. Microbiol. 33, 1402-1407 (1995).

23. Spence, R. P. et al. Population structure and antibiotic resistance of Acinetobacter DNA group 2 and 13TU isolates from hospitals in the UK. J. Med. Microbiol. 51, 1107-1112 (2002)

24. Lee, J. H. et al. Differences in phenotypic and genotypic traits against antimicrobial agents between Acinetobacter baumannii and Acinetobacter genomic species 13TU. J. Antimicrob. Chemother. 59, 633-639 (2007)

25. Jawad, A., Seifert, H., Snelling, A. M., Heritage, J. \& Hawkey, P. M. Survival of Acinetobacter baumannii on dry surfaces: comparison of outbreak and sporadic isolates. J. Clin. Microbiol. 36, 1938-1941 (1998)

26. van den Broek, P. J. et al. Epidemiology of multiple Acinetobacter outbreaks in the Netherlands during the period 1999-2001. Clin. Microbiol. Infect. 12, 837-843 (2006)

An analysis of eight concurrent outbreaks in one country and the measures that brought them under control.

27. Bernards, A. T. et al. Methicillin-resistant Staphylococcus aureus and Acinetobacter baumannil: an unexpected difference in epidemiologic behavior Am. J. Infect. Control 26, 544-551 (1998).

28. Dijkshoorn, L., Van Vianen, W., Degener, J. E. \& Michel, M. F. Typing of Acinetobacter calcoaceticus strains isolated from hospital patients by cell envelope protein profiles. Epidemiol. Infect. 99, 659-667 (1987).

29. Dijkshoorn, L. et al. Use of protein profiles to identify Acinetobacter calcoaceticus in a respiratory care unit. J. Clin. Pathol. 42, 853-857 (1989).

30. Nemec, A., Janda, L., Melter, O. \& Dijkshoorn, L. Genotypic and phenotypic similarity of multiresistant Acinetobacter baumannii isolates in the Czech Republic. J. Med. Microbiol. 48, 287-296 (1999).

31. Turton, J. F. et al. A prevalent, multiresistant clone of Acinetobacter baumannii in Southeast England. J. Hosp. Infect. 58, 170-179 (2004).

32. Da Silva, G. J., Dijkshoorn, L., van der Reijden, T., van Strijen, B. \& Duarte, A. Identification of widespread, closely related Acinetobacter baumannii isolates in Portugal as a subgroup of European clone II. Clin. Microbiol. Infect. 13, 190-195 (2007).

33. Quale, J., Bratu, S., Landman, D. \& Heddurshetti, R. Molecular epidemiology and mechanisms of carbapenem resistance in Acinetobacter baumannii endemic in New York City. Clin. Infect. Dis. 37, 214-220 (2003).

34. Dijkshoorn, L. et al. Comparison of outbreak and nonoutbreak Acinetobacter baumannii strains by genotypic and phenotypic methods. J. Clin. Microbiol. 34, 1519-1525 (1996)

35. Nemec, A., Dijkshoorn, L. \& van der Reijden, T. Long-term predominance of two pan-European clones among multi-resistant Acinetobacter baumannii strains in the Czech Republic. J. Med. Microbiol. 53, 147-153 (2004). A compilation of the properties of $A$. baumannii strains that belong to European clones I and II.

36. van Dessel, H. et al. Identification of a new geographically widespread multiresistant Acinetobacter baumannii clone from European hospitals. Res. Microbiol. 155, 105-112 (2004)

37. Seifert, H., Strate, A., Schulze, A. \& Pulverer, G Vascular catheter-related bloodstream infection due to Acinetobacter johnsonii (formerly Acinetobacter calcoaceticus var. Iwoffii): report of 13 cases. Clin. Infect. Dis. 17, 632-636 (1993)

38. Seifert, H., Strate, A., Schulze, A. \& Pulverer, C. Bacteremia due to Acinetobacter species other than Acinetobacter baumannii. Infection 22, 379-385 (1994).

39. Nemec, A. et al. Acinetobacter ursingii sp. nov. and Acinetobacter schindleri sp. nov., isolated from human clinical specimens. Int. J. Syst. Evol. Microbiol. 51, 1891-1899 (2001)

40. Nemec, A. et al. Acinetobacter parvus sp. nov., a small-colony-forming species isolated from human clinical specimens. Int. J. Syst. Evol. Microbiol. 53, 1563-1567 (2003)

41. de Beaufort, A. J., Bernards, A. T., Dijkshoorn, L. \& van Boven, C. P. Acinetobacter junii causes lifethreatening sepsis in preterm infants. Acta Paediatr 88, 772-775 (1999).

42. Garcia-Garmendia, J. L. et al. Risk factors for Acinetobacter baumannii nosocomial bacteremia in critically ill patients: a cohort study. Clin. Infect. Dis. 33 939-946 (2001).

43. Wisplinghoff, H., Perbix, W. \& Seifert, H. Risk factors for nosocomial bloodstream infections due to Acinetobacter baumannii: a case-control study of adult burn patients. Clin. Infect. Dis. 28, 59-66 (1999).

44. Maragakis, L. L. et al. An outbreak of multidrugresistant Acinetobacter baumannii associated with pulsatile lavage wound treatment. JAMA 292 3006-3011 (2004)

45. Cisneros, J. M. et al. Bacteremia due to Acinetobacter baumannii: epidemiology, clinical findings, and prognostic features. Clin. Infect. Dis. 22, 1026-1032 (1996)

46. Seifert, H., Strate, A. \& Pulverer, G. Nosocomial bacteremia due to Acinetobacter baumannii. Clinical features, epidemiology, and predictors of mortality. Medicine 74, 340-349 (1995).

47. Siegman-Igra, Y., Bar-Yosef, S., Gorea, A. \& Avram, J. Nosocomial acinetobacter meningitis secondary to invasive procedures: report of 25 cases and review. Clin. Infect. Dis. 17, 843-849 (1993).

48. Davis, K. A., Moran, K. A., McAllister, C. K. \& Gray, P. J. Multidrug-resistant Acinetobacter extremity infections in soldiers. Emerg. Infect. Dis. 11, 1218-1224 (2005). Important paper on Acinetobacter infections in wounded soldiers.

49. Wise, K. A. \& Tosolini, F. A. Epidemiological surveillance of Acinetobacter species. J. Hosp. Infect. 16, 319-329 (1990).

50. Blot, S., Vandewoude, K. \& Colardyn, F. Nosocomial bacteremia involving Acinetobacter baumannii in critically ill patients: a matched cohort study. Intensive Care Med. 29, 471-475 (2003).

51. Garnacho, J., Sole-Violan, J., Sa-Borges, M., Diaz, E. \& Rello, J. Clinical impact of pneumonia caused by Acinetobacter baumannii in intubated patients: a matched cohort study. Crit. Care Med. 31 . 2478-2482 (2003)

52. Falagas, M. E., Bliziotis, I. A. \& Siempos, I. I. Attributable mortality of Acinetobacter baumannii infections in critically ill patients: a systematic review of matched cohort and case-control studies. Crit. Care 10. R48 (2006)

53. Falagas, M. E. \& Rafailidis, P. I. Attributable mortality of Acinetobacter baumannii: no longer a controversial issue. Crit. Care 11, 134 (2007).

54. Anstey, N. M. et al. Community-acquired bacteremic Acinetobacter pneumonia in tropical Australia is caused by diverse strains of Acinetobacter baumannii, with carriage in the throat in at-risk groups. J. Clin. Microbiol. 40, 685-686 (2002).

55. Chen, M. Z. et al. Severe community-acquired pneumonia due to Acinetobacter baumannii. Chest 120, 1072-1077 (2001)

56. Oncul, O. et al. Hospital-acquired infections following the 1999 Marmara earthquake. J. Hosp. Infect. $\mathbf{5 1}$ 47-51 (2002)

57. Kennedy, P. J., Haertsch, P. A. \& Maitz, P. K. The Bali burn disaster: implications and lessons learned. J. Burn Care Rehabil. 26, 125-131 (2005).

58. Scott, P. et al. An outbreak of multidrug-resistant Acinetobacter baumannii-calcoaceticus complex infection in the US military health care system associated with military operations in Iraq. Clin. Infect. Dis. 44, 1577-1584 (2007)

59. Smith, M. G. et al. New insights into Acinetobacter baumannii pathogenesis revealed by high-density pyrosequencing and transposon mutagenesis. Genes Dev. 21, 601-614 (2007)

60. Wisplinghoff, H., Schmitt, R., Wohrmann, A. Stefanik, D. \& Seifert, H. Resistance to disinfectants in epidemiologically defined clinical isolates of Acinetobacter baumannii. J. Hosp. Infect. 66 174-181 (2007)

61. Tomaras, A. P., Dorsey, C. W., Edelmann, R. E. \& Actis, L. A. Attachment to and biofilm formation on abiotic surfaces by Acinetobacter baumannii: involvement of a novel chaperone-usher pili assembly system. Microbiology 149, 3473-3484 (2003).

62. Lee, J. C. et al. Adherence of Acinetobacter baumannil strains to human bronchial epithelial cells. Res. Microbiol. 157, 360-366 (2005).

63. Haseley, S. R., Pantophlet, R., Brade, L., Holst, O. \& Brade, H. Structural and serological characterisation of the O-antigenic polysaccharide of the lipopolysaccharide from Acinetobacter junii strain 65. Eur. J. Biochem. 245, 477-481 (1997)

64. Choi, C. H. et al. Acinetobacter baumannii outer membrane protein A targets the nucleus and induces cytotoxicity Cell Microbiol. 30 Aug 2007 (doi: 10.1111/j.1462-5822.2007.01041.x)

65. Dorsey, C. W., Beglin, M. S. \& Actis, L. A. Detection and analysis of iron uptake components expressed by Acinetobacter baumannii clinical isolates. J. Clin. Microbiol. 41, 4188-4193 (2003).

66. Jankowski, S., Grzybek-Hryncewicz, K., Fleischer, M. \& Walczuk, M. Susceptibility of isolates of Acinetobacter anitratus and Acinetobacter Iwoffii to the bactericidal activity of normal human serum. FEMS Microbiol. Immunol. 4, 255-260 (1992).

67. Brade, H. \& Galanos, C. Biological activities of the lipopolysaccharide and lipid A from Acinetobacter calcoaceticus. J. Med. Microbiol. 16, 211-214 (1983).

68. Knapp, S. et al. Differential roles of CD14 and toll-like receptors 4 and 2 in murine Acinetobacter pneumonia. Am. J. Respir. Crit Care Med. 173 122-129 (2006)

69. Perez, F. et al. Global challenge of multidrug-resistant Acinetobacter baumannii. Antimicrob. Agents Chemother. 51, 3471-3484 (2007). State-of-the-art review on MDR A. baumannii.

70. Vila, J. et al. In vitro antimicrobial production of $\beta$-lactamases, aminoglycoside-modifying enzymes, and chloramphenicol acetyltransferase by and susceptibility of clinical isolates of Acinetobacter baumannii. Antimicrob. Agents Chemother. 37 , 138-141 (1993)

71. Seifert, H., Baginski, R., Schulze, A. \& Pulverer, G. Antimicrobial susceptibility of Acinetobacter species. Antimicrob. Agents Chemother. 37, 750-753 (1993).

72. Seifert, H., Stefanik, D. \& Wisplinghoff, H Comparative in vitro activities of tigecycline and 11 other antimicrobial agents against 215 epidemiologically defined multidrug-resistant Acinetobacter baumannii isolates. J. Antimicrob. Chemother. 58, 1099-1100 (2006).

73. Tankovic, J. et al. Characterization of a hospital outbreak of imipenem-resistant Acinetobacter baumannii by phenotypic and genotypic typing methods. J. Clin. Microbiol. 32, 2677-2681 (1994).

74. Turner, P. J. \& Greenhalgh, J. M. The activity of meropenem and comparators against Acinetobacter strains isolated from European hospitals, 1997-2000. Clin. Microbiol. Infect. 9, 563-567 (2003).

75. Li, J. et al. Heteroresistance to colistin in multidrugresistant Acinetobacter baumannii. Antimicrob. Agents Chemother. 50, 2946-2950 (2006).

76. Peleg, A. Y. et al. Acinetobacter baumanni bloodstream infection while receiving tigecycline: a cautionary report. J. Antimicrob. Chemother. 59, 128-131 (2007)

77. Seward, R. J., Lambert, T. \& Towner, K. J. Molecular epidemiology of aminoglycoside resistance in Acinetobacter spp. J. Med. Microbiol. 47, 455-462 (1998).

78. Doi, Y et al Spread of novel aminoglycoside resistance gene aac (6')-lad among Acinetobacter clinical isolates in Japan. Antimicrob. Agents Chemother. 48, 2075-2080 (2004).

79. Vila, J., Ruiz, J., Goni, P. \& Jimenez de Anta, T. Quinolone-resistance mutations in the topoisomerase IV parC gene of Acinetobacter baumannii. J. Antimicrob. Chemother. 39, 757-762 (1997).

80. Huys, G. et al. Distribution of tetracycline resistance genes in genotypically related and unrelated multiresistant Acinetobacter baumannii strains from different European hospitals. Res. Microbiol. 156 348-355 (2005)

81. Heritier, C., Poirel, L. \& Nordmann, P. Cephalosporinase over-expression resulting from insertion of ISAba 1 in Acinetobacter baumannii. Clin. Microbiol. Infect. 12, 123-130 (2006)

82. Livermore, D. M. $\&$ Woodford, N. The $\beta$-lactamase threat in Enterobacteriaceae, Pseudomonas and Acinetobacter. Trends Microbiol. 14, 413-420 (2006).

83. Turton, J. F. et al. The role of ISAba 1 in expression of OXA carbapenemase genes in Acinetobacter baumannii. FEMS Microbiol. Lett. 258, 72-77 (2006).

84. Magnet, S., Courvalin, P. \& Lambert, T. Resistance-nodulation-cell division-type efflux pump involved in aminoglycoside resistance in Acinetobacter baumannii strain BM4454. Antimicrob. Agents Chemother. 45, 3375-3380 (2001).

85. Marchand, I., Damier-Piolle, L., Courvalin, P. \& Lambert, T. Expression of the RND-type efflux pump 
Ade $A B C$ in Acinetobacter baumannii is regulated by the AdeRS two-component system. Antimicrob. Agents Chemother. 48, 3298-3304 (2004).

86. Poirel, L. \& Nordmann, P. Carbapenem resistance in Acinetobacter baumannii: mechanisms and epidemiology. Clin. Microbiol. Infect. 12, 826-836 (2006).

State-of-the-art review on the mechanisms that are associated with the carbapenem resistance of A. baumannii.

87 Bou, G. Cervero, G., Dominguez, M. A., Quereda, C. \& Martinez-Beltran, J. Characterization of a nosocomial outbreak caused by a multiresistant Acinetobacter baumannii strain with a carbapenemhydrolyzing enzyme: high-level carbapenem resistance in A. baumannii is not due solely to the presence of ß-lactamases. J. Clin. Microbiol. 38, 3299-3305 (2000).

88. Nemec, A., Dolzani, L., Brisse, S., Van Den, B. P. \& Dijkshoorn, L. Diversity of aminoglycoside-resistance genes and their association with class 1 integrons among strains of pan-European Acinetobacter baumannii clones. J. Med. Microbiol. 53, 1233-1240 (2004).

89 Corbella, X et al. Efficacy of sulbactam alone and in combination with ampicillin in nosocomial infections caused by multiresistant Acinetobacter baumannii. J. Antimicrob. Chemother. 42, 793-802 (1998).

90. Wood, G. C., Hanes, S. D., Croce, M. A., Fabian, T. C. \& Boucher, B. A. Comparison of ampicillin-sulbactam and imipenem-cilastatin for the treatment of acinetobacter ventilator-associated pneumonia. Clin Infect. Dis. 34, 1425-1430 (2002).

91. Garnacho-Montero, J. et al. Treatment of multidrugresistant Acinetobacter baumannii ventilatorassociated pneumonia (VAP) with intravenous colistin: a comparison with imipenem-susceptible VAP. Clin. Infect. Dis. 36, 1111-1118 (2003)

92. Kwa, A. L., Loh, C., Low, J. G., Kurup, A. \& Tam, V. H. Nebulized colistin in the treatment of pneumonia due to multidrug-resistant Acinetobacter baumannii and Pseudomonas aeruginosa. Clin. Infect. Dis. 41, 754-757 (2005)

93. Navon-Venezia, S., Leavitt, A. \& Carmeli, Y. High tigecycline resistance in multidrug-resistant Acinetobacter baumannii. J. Antimicrob. Chemother. 59, 772-774 (2007)

94. Petrosillo, N. et al. Combined colistin and rifampicin therapy for carbapenem-resistant Acinetobacter baumannii infections: clinical outcome and adverse events. Clin. Microbiol. Infect. 11, 682-683 (2005).

95. Saballs, M. et al. Rifampicin/imipenem combination in the treatment of carbapenem-resistant Acinetobacter baumannii infections. J. Antimicrob. Chemother. 58 697-700 (2006)

96. Pantopoulou, A. et al. Colistin offers prolonged survival in experimental infection by multidrug resistant Acinetobacter baumannii: the significance of co-administration of rifampicin. Int. J. Antimicrob. Agents 29, 51-55 (2007)

97. Montero, A. et al. Antibiotic combinations for serious infections caused by carbapenem-resistant Acinetobacter baumannii in a mouse pneumonia model. J. Antimicrob. Chemother. 54, 1085-1091 (2004)

98. Dijkshoorn, L. et al. The synthetic N-terminal peptide of human lactoferrin, $h L F(1-11)$, is highly effective against experimental infection caused by multidrugresistant Acinetobacter baumannii. Antimicrob. Agents Chemother. 48, 4919-4921 (2004).

99. Dijkshoorn, L., van Harsselaar, B., Tjernberg, I., Bouvet, P. J. \& Vaneechoutte, M. Evaluation of amplified ribosomal DNA restriction analysis for identification of Acinetobacter genomic species. Syst. Appl. Microbiol. 21, 33-39 (1998).

100. Vaneechoutte, M. et al. Naturally transformable Acinetobacter sp. strain ADP1 belongs to the newly described species Acinetobacter baylyi. Appl. Environ Microbiol. 72, 932-936 (2006).

101. La Scola, B., Gundi, V. A., Khamis, A. \& Raoult, D. Sequencing of the $r p o B$ gene and flanking spacers for molecular identification of Acinetobacter species. J. Clin. Microbiol. 44, 827-832 (2006).

102. Bernards, A. T., Harinck, H. I., Dijkshoorn, L., van der Reijden, T. \& van den Broek, P. J. Persistent Acinetobacter baumannii? Look inside your medical equipment. Infect. Control Hosp. Epidemiol. 25 1002-1004 (2004)

103. Villegas, M. V. \& Hartstein, A. I. Acinetobacter outbreaks, 1977-2000. Infect. Control Hosp. Epidemiol. 24, 284-295 (2003)

104. Weernink, A., Severin, W. P., Tjernberg, I. \& Dijkshoorn, L. Pillows, an unexpected source of Acinetobacter. J. Hosp. Infect. 29, 189-199 (1995).

105. Nishimura, Y., Ino, T. \& lizuka, H. Acinetobacter radioresistens $\mathrm{sp}$. nov. isolated from cotton and soil. Int. J. Syst. Bacteriol. 38, 209-211 (1988).

106. Carr, E. L., Kämpfer, P., Patel, B. K., Gurtler, V. \& Seviour, R. J. Seven novel species of Acinetobacter isolated from activated sludge. Int. J. Syst. Evol. Microbiol. 53, 953-963 (2003).

107. Vaneechoutte, M. et al. Oil-degrading Acinetobacter strain RAG-1 and strains described as Acinetobacter venetianus sp. nov.' belong to the same genomic species. Res. Microbiol. 150, 69-73 (1999)

108. Bouvet, P. J. \& Jeanjean, S. Delineation of new proteolytic genomic species in the genus Acinetobacter. Res. Microbiol. 140, 291-299 (1989)

09. Hujer, K. M. et al. Identification of a new allelic variant of the Acinetobacter baumannii cephalosporinase ADC-7 $\beta$-lactamase: defining a unique family of class $C$ enzymes. Antimicrob. Agents Chemother 49, 2941-2948 (2005)

110. Devaud, M., Kayser, F. H. \& Bachi, B. Transposonmediated multiple antibiotic resistance in Acinetobacter strains. Antimicrob. Agents Chemother 22, 323-329 (1982).

111. Paul, G., Joly-Guillou, M. L., Bergogne-Bérézin, E., Nevot, P. \& Philippon, A. Novel carbenicillin hydrolyzing $\beta$-lactamase (CARB-5) from Acinetobacter calcoaceticus var. anitratus. FEMS Microbiol. Lett. $\mathbf{5 0}$ 45-50 (1989).

112. Poirel, L. et al. Identification of the novel narrowspectrum $\beta$-lactamase SCO- 1 in Acinetobacter spp. from Argentina. Antimicrob. Agents Chemother. $\mathbf{5 1}$ 2179-2184 (2007)

113. Vila, J., Navia, M., Ruiz, J. \& Casals, C. Cloning and nucleotide sequence analysis of a gene encoding an OXA-derived $\beta$-lactamase in Acinetobacter baumannii. Antimicrob. Agents Chemother. $\mathbf{4 1}$ 2757-2759 (1997)

114. Navia, M. M., Ruiz, J. \& Vila, J. Characterization of an integron carrying a new class D $\beta$-lactamase (OXA-37) in Acinetobacter baumannii. Microb. Drug Resist. 8, 261-265 (2002).

115 Lambert, T Gerbaud, G \& Courvalin, P. Characterization of the chromosomal $\operatorname{aac}\left(6^{\prime}\right)-l j$ gene of Acinetobacter sp. 13 and the aac (6')-Ih plasmid gene of Acinetobacter baumannii. Antimicrob. Agents Chemother. 38, 1883-1889 (1994).

116. Lambert, T., Gerbaud, G. \& Courvalin, P. Transferable amikacin resistance in Acinetobacter spp. due to a new type of $3^{\prime}$-aminoglycoside phosphotransferase. Antimicrob. Agents Chemother 32, 15-19 (1988)

117. Lee, H. et al. Dissemination of 16S rRNA methylase mediated highly amikacin-resistant isolates of Klebsiella pneumoniae and Acinetobacter baumannii in Korea. Diagn. Microbiol. Infect. Dis. 56, 305-312 (2006).

118. Su, X. Z., Chen, J., Mizushima, T., Kuroda, T. \& Tsuchiya, T. AbeM, an $\mathrm{H}^{+}$-coupled Acinetobacter baumannii multidrug efflux pump belonging to the MATE family of transporters. Antimicrob. Agents Chemother. 49, 4362-4364 (2005).

\section{Acknowledgements}

A.N. has been supported by grant NR 8554-3 from the Internal Grant Agency of the Ministry of Health of the Czech Republic. The authors thank P. van den Broek for critical reading of the manuscript and helpful discussions and are grateful to T. van der Reijden and other members of their laboratories for their support.

\section{DATABASES}

Entrez Genome Project: http://www.ncbi.nlm.nih.gov/ entrez/query.fcgi?db=genomeprj Acinetobacter baumannii| Pseudomonas aeruginosa | Staphylococcus aureus

Entrez Protein: http://www.ncbi.nlm.nih.gov/entrez/query. fcgi?db=protein

AbOmpA

\section{FURTHER INFORMATION}

Acinetobacter baumannii and other pathogens in injured soldiers: http://www.acinetobacter.org

Acinetobacter baumannii MLST database Institut Pasteur, Paris, FR: http://www.pasteur.fr/recherche/genopole/PF8/ mlst/Abaumannii.htm

Acinetobacter baumannii MLST databases University

Oxford, UK: http://pubmlst.org/abaumannii

ARDRA for Acinetobacter species identification: http:// users.ugent.be/ mvaneech/ARDRA/Acinetobacter.html Dutch working party on infection prevention (WIP): http://www.wip.nl/UK

ALL LINKS ARE ACTIVE IN THE ONLINE PDF 